\title{
Artefact
}

Techniques, histoire et sciences humaines

2 | 2014

Art et industrie : les enjeux de la formation (XVIII $\mathrm{XX}$ ' siècles) / Qu'est-ce qu'un outil simple?

\section{Former les jeunes au dessin sur support céramique : capitalisation des connaissances et action sociale au sein de la fabrique Collinot \& $C^{\text {ie }}$}

\section{Sabine Pasdelou}

\section{(2) OpenEdition}

Journals

Édition électronique

URL : https://journals.openedition.org/artefact/8919

DOI : $10.4000 /$ artefact.8919

ISSN : 2606-9245

Éditeur :

Association Artefact. Techniques histoire et sciences humaines, Presses universitaires du Midi

Édition imprimée

Date de publication : 11 septembre 2014

Pagination : $43-59$

ISBN : 978-2-271-08150-6

ISSN : 2273-0753

\section{Référence électronique}

Sabine Pasdelou, «Former les jeunes au dessin sur support céramique : capitalisation

des connaissances et action sociale au sein de la fabrique Collinot \& Cie », Artefact [En ligne], 2 | 2014 mis en ligne le 11 mai 2021, consulté le 24 août 2021. URL : http://journals.openedition.org/artefact/ 8919 ; DOI : https://doi.org/10.4000/artefact.8919

\section{(c) (i) (9)}

Artefact, Techniques, histoire et sciences humaines est mise à disposition selon les termes de la Licence Creative Commons Attribution - Pas d'Utilisation Commerciale - Pas de Modification 4.0 International. 


\section{Former les jeunes au dessin sur support céramique : capitalisation des connaissances et action sociale au sein de la fabrique Collinot \& $C^{i e}$}

Sabine PASDELOU ${ }^{1}$

\section{Résumé}

Cette étude porte sur la collaboration entre l'ornemaniste-théoricien Adalbert de Beaumont et le céramiste Eugène Collinot, qui fondent une faïencerie en 1863. Les deux hommes transmettent leurs connaissances selon des projets qui correspondent à leur sensibilité et à leurs compétences techniques en s'adressant à des publics variés. Beaumont redéfinit les fonctionnalités de l'ornement et du dessin selon un schème social issu de ses théories sur les arts décoratifs. La souscription de son recueil d'ornements par l'État lui permet de transmettre ses modèles à plusieurs écoles d'art industriel de province. Collinot se concentre quant à lui sur la formation locale de jeunes apprentis orphelins ou indigents qu'il initie au dessin sur support céramique au sein de son "école d'apprentissage mutuel de l'enfance pour la céramique", créée en 1867. Cet article met ainsi l'accent sur les enjeux sociaux de leurs projets qui s'inscrivent dans un contexte de revivification de l'art industriel français.

Mots-clés : apprentissage mutuel, art industriel, atelier de décoration, céramique, dessin, recueil d'ornements.

\section{Abstract}

This study focuses on the collaboration between the decorator-theorist Adalbert de Beaumont and the ceramist Eugene Collinot who founded an earthenware factory in 1863. The two men transmitted their knowledge according to their projects that matched their sensitivity and their technical skills while being addressed to a variety of audiences. Beaumont redefined the functionality of the ornament and the drawing according to a social scheme resulting from his theories on decorative arts. The subscription of its pattern book 
by the state allowed it to transmit its models in several industrial art schools of province. Collinot focused on the local training of young orphan or poor apprentices whom he initiated with the drawing on ceramic support within its "School of mutual training of childhood for ceramics", created in 1867. So, this article stresses the social issues in their projects that fit within the context of the revival of French industrial art.

Keywords: ceramic decoration, decoration workshop, drawing, industrial art, mutual training, pattern book.

À la suite de l'Exposition internationale de 1851, à Londres, le comte Léon de Laborde ${ }^{2}$ s'interroge, dans son volumineux rapport si souvent cité, sur l'industrialisation de l'art et le rôle de l'État dans la formation des artistes ${ }^{3}$. Bien que certains auteurs refusent ses propositions, comme le critique littéraire Gustave Planche, Laborde trouve un écho très favorable auprès de certains critiques, comme Adalbert de Beaumont (1809-1869).

Rien ne semble prédestiner ce jeune aristocrate à une carrière industrielle. En 1833, Beaumont débute un périple en Russie, avant de séjourner à plusieurs reprises en Orient et dans le bassin méditerranéen entre 1834 et $1845^{4}$. Il remplit des carnets de croquis dans lesquels il réalise différents paysages et montre un vif intérêt pour la nature. Artiste-voyageur, il se forme lors de ses voyages en Italie et en Orient et commence sa carrière comme peintre de Salon et littérateur. Déçu par son environnement artistique, il décide de se consacrer à la rédaction de critiques interrogeant le devenir de l'art industriel $l^{5}$. Il édite des planches d'ornements, issues pour la plupart de ses voyages et de ses rencontres avec des collectionneurs et qui forment le Recueil pour l'art et l'industrie (1859-1873). De tels recueils d'ornements et périodiques d'art se multiplient depuis le début du XIX ${ }^{\mathrm{e}}$ siècle. Les lois sur la liberté de la presse et les améliorations techniques d'impression à faible coût ne sont pas étrangères à cette large diffusion ${ }^{6}$. Ces publications sont aussi des soutiens à l'apprentissage dont Beaumont juge le rôle crucial pour l'art industriel.

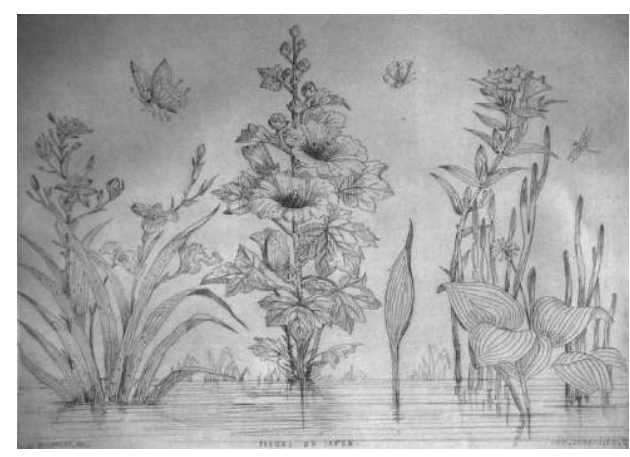

Fig. 1 - Adalbert de Beaumont, « Fleurs du Japon », planche 200, Recueil pour l'art et l'industrie, volume II, 1867, 27x18 cm, gravure à l'eau-forte, Longwy, Institut de formation céramographique Saint-Jean-l'Aigle, CInstitut Saint-Jean-l'Aigle, Longwy. 


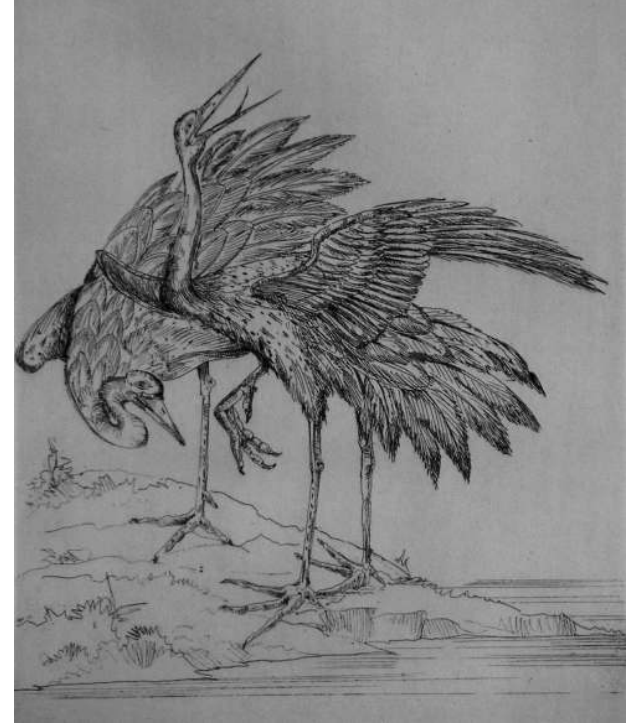

Fig. 2 - Adalbert de Beaumont, « Oiseaux japonais ", planche 191, Recueil pour l'art et l'industrie, volume II, 1866, 18x15 cm, gravure à l'eau-forte, Longwy, Institut de formation céramographique Saint-Jean-l'Aigle, @Institut Saint-Jean-l'Aigle, Longwy.

Néanmoins, l'action et la réflexion de Beaumont restent plus largement celles d'un entrepreneur aux capacités multiples qu'il sait transmettre, que le support soit discursif, graphique ou céramique. En effet, en collaboration avec Eugène-Victor Collinot (1824-1889), militaire de carrière et «chimiste praticien ${ }^{7}$ », il s'investit dans une fabrique de faïences d'art. Dès le départ, les rôles sont définis et Beaumont initie Collinot à l'ornement appliqué à la céramique. En conséquence, Beaumont peut poursuivre ses publications, tandis que son collaborateur supervise le fonctionnement de la manufacture et s'occupe de la formation des apprentis-élèves.

D'un projet initialement centré sur la formation d'une main-d'œuvre locale jeune et inexpérimentée, la collaboration des deux hommes devient un projet global, sous-tendu par des questions sociales en lien avec les métiers d'art. Alors que de nombreuses études portant sur la céramique indépendante insistent sur la biographie et le rôle des protagonistes ${ }^{8}$, nous optons pour une recontextualisation globale des actions artistiques et sociales des deux hommes aux sensibilités et ambitions propres ${ }^{9}$. Dans un premier temps, nous nous intéresserons aux premières manifestations $\mathrm{du}$ projet chez Collinot \& $C^{\text {ie }}$ et aux idéaux sociaux qui le sous-tendent. Aussi, nous faut-il, dans une seconde partie, décrire la mise en place de l'école et, surtout, nous interroger sur les retombées de la formation. Enfin, il s'agit d'inscrire les tentatives de Beaumont et de Collinot dans un contexte de revivification des arts industriels français.

\section{Collinot $\& \mathrm{C}^{\mathrm{ie}}$ : coopération et diffusion des connaissances de la pratique céramique artistique (1859-1889)}

Adalbert de Beaumont rédige une série d'articles entre 1848 et 1866, rapportant ses impressions de voyage, ses préceptes sur l'art décoratif oriental et ses cri- tiques de l'art français contemporain ${ }^{10}$. À la suite de la publication de Laborde, Beaumont engage une réflexion sur ce débat $^{11}$ et sur le rôle que devrait exercer 


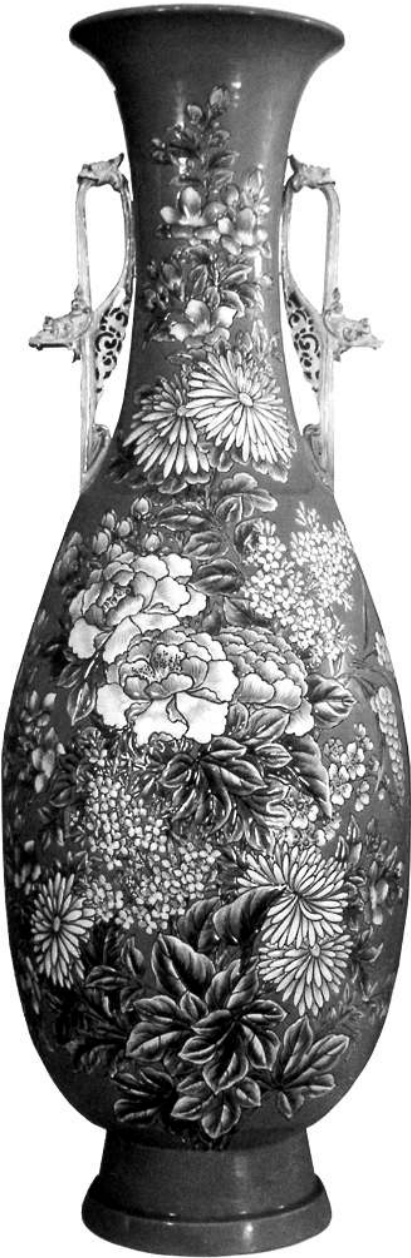

Fig. 3 - Eugène Collinot, Vase monumental, faïence à pâte chamottée blanche, émaux en relief cerné, circa 1867, 103 cm, Longwy, Institut de formation céramographique Saint-Jean-l'Aigle,

CInstitut Saint-Jean-l'Aigle, Longwy.

l'art oriental et asiatique sur l'art industriel français ${ }^{12}$. L'idéal de Laborde dans la réconciliation de l'art et de l'industrie est alors considéré comme une provocation auprès des critiques en raison $\mathrm{du}$ pragmatisme des industries d'art et, plus particulièrement, à cause de la notion de profit et de la fabrication massive

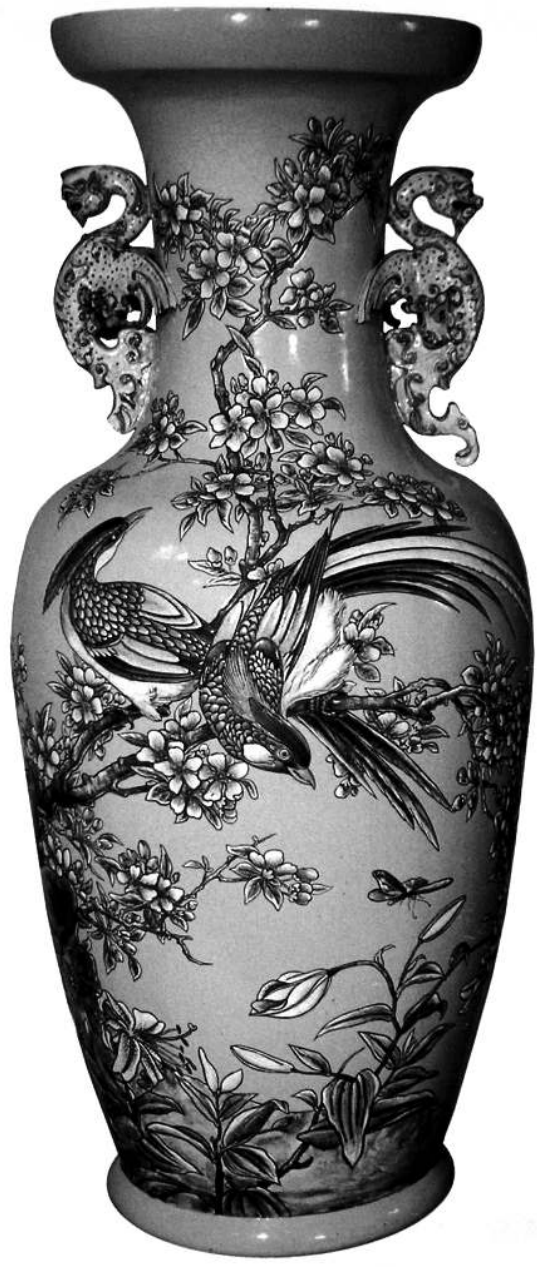

Fig. 4 - Eugène Collinot, Vase monumental d'une paire, faïence à pâte chamottée blanche, émaux en relief cerné, circa 1868-1878, 92 cm, Longwy, Institut de formation céramographique Saint-Jeanl'Aigle, OInstitut Saint-Jean-l'Aigle, Longwy.

d'objets, incompatibles avec la notion $d^{\prime} œ u v r e d^{\prime} \operatorname{art}^{13}$. Beaumont adhère pleinement au principe d'alliance entre l'art et l'industrie. La situation artistique en France, qu'il juge décadente et sclérosée, souffrirait du désengagement de l'Empire dans les actions en faveur de l'art industriel, favorisant ainsi une forme 
de matérialisme dénué de sentiment artistique $^{14}$. Une de ses propositions est donc de modifier la formation technique à l'échelle nationale car l'«enseignement de l'art industriel est à réformer complètement ${ }^{15}$ ». L'auteur souhaite transmettre les connaissances acquises au cours de ses voyages en les intégrant à une démarche critique, historique et pédagogique. Alors que les théoriciens des arts décoratifs sont attentifs aux découvertes archéologiques et ethnographiques, la littérature de Beaumont est imprégnée de son admiration pour la culture et les arts orientaux, se risquant souvent à faire des analogies avec les arts français.

Avant sa collaboration avec Eugène Collinot, Beaumont s'est déjà intéressé à la céramique. Bien que sa formation à cet art soit inconnue, il semble le pratiquer très tôt. En 1845, l'atelier qu'il a dans l'appartement de sa mère ne lui convient plus $^{16}$. En 1859, il mentionne sa charge de décorateur de certains moules intermédiaires ${ }^{17}$. Beaumont expose dans un premier temps avec les frères Deck des pièces de style oriental lors de l'exposition de la Société du progrès pour l'art industriel du Palais de l'industrie de 1861 ${ }^{18}$. À partir de 1863, Collinot expose «avec le concours artistique de M. Adalbert de Beaumont ${ }^{19}$ » lors de la première exposition de l'Union centrale des beauxarts appliqués à l'industrie ${ }^{20}$. Beaumont et Collinot se seraient rencontrés en 1855 et, quatre ans plus tard, débute une collaboration qui ne cesse qu'à la mort de Beaumont ${ }^{21}$. Les premières années de leur coopération furent consacrées essentiellement à la formation de Collinot par Beaumont: «M. Collinot a acheté un four, et fait fabriquer d'après mes enseignements $^{22}$.» Dès 1859, ils réalisent des céramiques qui, comme il le déplore auprès de son ami Charles Cournault, «vont leur train mais lentement ${ }^{23}$ ». Leur atelier parisien provisoire, situé d'abord avenue de Saxe, déménage chez Beaumont, avenue de la Porte Maillot, où il ne réalise que ses gravures et ses articles. En effet, au début de cette coopération, chacun reste affecté à ses tâches en raison du manque de place et de l'insuffisance de leurs moyens techniques: les deux hommes ne disposent pas d'un atelier commun (1861) ni d'un four (1862). Après avoir transmis ses connaissances à Collinot, Beaumont se considère comme le superviseur artistique et créateur de modèles: "J'ai reçu de mon faïencier [...] un vase arabe nankin et bleu de trois tons qui a bien réussi. Les reliefs sont bien exécutés, enfin nous nous sommes donné de la peine, mais pas inutilement; car c'est moi qui ai gravé sur le moule tout le dessin, il n'a eu qu'à évider et à cuire $^{24}$.» Leur établissement définitif à Boulogne-sur-Seine, avenue du Parc des Princes, leur permettra de se stabiliser et d'augmenter leurs ressources. Alors que Beaumont aborde les objets sous leur angle esthétique, Collinot développe plutôt ses compétences pratiques. L'alliance entre les deux hommes, dont les orientations semblent au premier abord opposées, forme le juste équilibre entre l'objet céramique et sa charge esthétique. La contribution de Beaumont décroît au fur et à mesure que l'apprentissage de Collinot s'achève. Ce dernier aide à la réalisation des pièces de forme, des céramiques architecturales, ainsi qu'à la décoration des pièces. Les grandes pièces sont réalisées au sein de la fabrique de faïence de Toul-Bellevue car, comme l'explique Beaumont, «nous 
ne faisons que les petites pièces, nous ne sommes pas encore montés pour cela $\mathrm{a}^{25}$ ». Les commandes auprès de cette fabrique prennent fin en 1863, année de l'édification du second four.

Beaumont est un technicien et un critique dont la sensibilité et les ambitions pédagogiques se diffusent grâce au Recueil pour l'art et l'industrie ${ }^{26}$, somme de ses connaissances artistiques. Il ne réalise pas moins de 249 eaux-fortes monochromes, dispersées sans classification précise, reproduisant différentes œuvres typiques de plusieurs civilisations (annexe 1 et Fig. 1 et 2). Le recueil obéit à une volonté de transmission de l'éducation artistique. En ce sens, il s'inscrit dans le développement de l'instruction, qui prend aussi la forme des bibliothèques populaires, et dans une intention d'édification par l'art. La démarche a débuté au XviII ${ }^{\mathrm{e}}$ siècle avec la création et le développement des écoles gratuites de desin ${ }^{27}$, puis, surtout, la loi sur les écoles centrales qui autorise les villes à ouvrir des bibliothèques. Depuis une ordonnance royale du 27 mars 1828, plusieurs bibliothèques publiques sont pourvues d'ouvrages préalablement déposés au dépôt légal et au ministère de $1^{\prime}$ Instruction publique ${ }^{28}$. Certains de ces ouvrages obtiennent une souscription de l'État. Les envois sont d'ailleurs effectués en fonction des entreprises situées aux alentours de la bibliothèque ou des écoles communales d'art ${ }^{29}$. En faisant parvenir ces livres, les responsables souhaitent améliorer la formation des ouvriers d'art. Le ministère de la Maison de l'Empereur et des Beaux-Arts établit une souscription à l'ouvrage de Beaumont entre 1864 et 1870, diffusant ainsi ses modèles dans plusieurs écoles d'art industriel de province (annexe 2).

Tandis que Beaumont poursuit ses activités éditoriales à travers ses théories et par la création d'un outil à destination des écoles d'art industriel de province, Collinot se concentre sur l'enseignement au sein de la fabrique.

\section{Une «École d'apprentissage mutuel de l'enfance pour la céramique ${ }^{30} »$}

Depuis son établissement à Boulogne, l'entreprise a pu augmenter ses effectifs. Elle rétribue une vingtaine d'employés en 1863: l'équipe s'affaire à la préparation de la prochaine exposition du Palais de l'Industrie. C'est un succès: «M. Collinot a été un de ceux qui ont le plus contribué à enlever la faïencerie artistique de l'état d'abaissement où l'avaient trouvée les expositions de 1851 et de $1855^{31}$.» Le dynamisme de l'entreprise, l'habileté technique et l'intensité des couleurs de leurs produits valent aux deux hommes chacun une médaille: Beaumont est récompensé pour l'originalité de ses modèles et pour avoir remis à l'honneur le goût de l'Orient dans les arts décoratifs, Collinot pour la qualité technique de ses œuvres. Le 30 septembre 1864, ses recherches se concrétisent par le dépôt et la délivrance d'un brevet d'invention $q^{\prime}$ 'il nomme «émail cloisonnés2 ${ }^{32}$. Cette 
appellation est fausse dans sa définition technique car il s'agit de la superposition d'émaux, fixés grâce à un fondant et délimités grâce à un cerne de pigment à base d'oxyde ${ }^{33}$ (Fig. 3 et 4 ). Dès lors, Collinot ne s'intéresse pas à la multiplication des découvertes techniques. Il préfère s'attacher à pérenniser ses émaux qu'il transmet à tous ses collaborateurs. Il refuse l'utilisation de machines pour la décoration des faïences, privilégiant le travail manuel. Ainsi, l'ouverture, en 1867, d'une école d'apprentissage à destination des enfants démunis ou orphelins prend tout son sens. Son intérêt est d'ailleurs sans doute en lien avec la récente création de la Société de protection des apprentis et des enfants des manufactures $(1866)^{34}$, qui souhaite améliorer la condition morale et sociale des jeunes apprentis. Son organe principal est son bulletin bimestriel ${ }^{35}$ dans lequel elle publie les rapports des conditions de travail des enfants et propose ses réflexions pour une application concrète des lois sur le travail des enfants. Ces questions intéressent Collinot: lui-même orphelin à l'âge de 12 ans, il est adopté par son oncle, le général Isidore Didion. Collinot choisit d'orienter son action à destination des enfants démunis. En tant que membre de la Société de protection des apprentis et des enfants des manufactures, il veille à appliquer les lois relatives au travail des enfants ${ }^{36}$.

Dans les divers documents requis pour l'obtention de la Légion d'honneur ${ }^{37}$, il est question de cette nouvelle institution à vocation formatrice, en activité depuis 1867: «Sa fabrique [...] est un bienfait pour le pays au milieu duquel il choisit des ouvriers qui dès 15 ans lorsqu'ils savent tenir un crayon gagneraient de suite leur vie, après un mois d'apprentissage ${ }^{38}$.» Selon ces documents, dont les propos ont été confirmés par le rapport de Paul Fouchet $^{39}$ cité dans le Bulletin de la Société de protection des apprentis et des enfants des manufactures, cette école permet à un certain nombre d'adolescents, âgés de 12 à 18 ans, de se former au métier de décorateur ${ }^{40}$. Selon Fouchet, Collinot applique un ordre de travail très régulier sans négliger les personnalités de ses jeunes employés et crée ainsi un emploi pour chacun. Les apprentis travaillent principalement dans l'atelier de décor. Collinot ou Beaumont se charge de la composition du décor et reporte le motif sur la céramique, laissant aux apprentis le soin d'épaissir les lignes de l'ornement avec un pinceau ou un autre instrument. Selon le rapporteur, c'est un «travail qui les intéresse et dans lequel ils acquièrent rapidement la sûreté de main nécessaire ${ }^{41}$ ». L'apprentissage du geste, à travers la pratique du dessin sur céramique, l'emporte sur l'aspect ludique mis en avant par Fouchet. L'auteur est très favorable au caractère progressif du rôle de l'enfant qui devient un futur formateur. L'évolution de l'apprenti est possible grâce à une compréhension de l'instruction de son supérieur et à la faculté à former lui-même un apprenti novice. Dans cet atelier, les jeunes apprennent à se former et à s'aider les uns les autres grâce à un système de tutorat. Par l'entraide, les aînés aident les plus jeunes. Si cette coopération fonctionne, les deux apprentis peuvent passer à une fonction supérieure. Cette question de l'enseignement mutuel est très vivace au $\mathrm{XIX}^{\mathrm{e}}$ siècle $^{42}$. Les responsabilités 
croissantes des apprentis suivent une évolution concentrique en quatre étapes et en adéquation avec l'augmentation de leur salaire: "chaque enfant est à la fois élève et moniteur: élève d'un plus fort et moniteur d'un plus faible que lui ${ }^{43}$.» Ils gagnent au minimum 1 franc par jour et peuvent atteindre 4 francs pour une journée de dix heures, la première année. La deuxième année, l'apprenti gagne au moins 4 francs par jour, la troisième 5 francs et la quatrième 6 francs $^{44}$. À la fin de cet apprentissage, les adolescents gagnent un salaire d'employé adulte et sont aptes à travailler dans différentes manufactures ou à créer leur atelier. Le dessein de Collinot n'est pas uniquement de former ses futurs collaborateurs; il conçoit la formation de ces jeunes gens comme nécessaire à leur carrière à la sortie de l'atelier ${ }^{45}$. Dans son rapport, Fouchet confirme le fait que les jeunes reçoivent un salaire proche de celui des adultes pendant leur formation à la production d'œuvres d'art qui sont présentées à l'exposition universelle de 1867. Collinot exploite les capacités de cette équipe: les pièces sont vendues à côté des chefs-d'œuvre de la fabrique au dépôt situé rue Royale, à Paris, où on peut voir des «vases de quarante mille francs devant lesquels on demeure en extase, et à côté des petits objets d'une valeur insignifiante, délicieusement jolis avec des fleurs en reliefs $[\ldots]^{46} »$. Il envisage par ailleurs la création d'un internat. En effet, il s'inquiète de l'errance des enfants ou de l'éventuel détournement de l'argent par la famille et souhaite offrir un toit ainsi qu'une éducation à ces jeunes. Il loge chez lui quelques apprentis et les emmène aux cours du soir dans les écoles primaires de Boulogne ${ }^{47}$, le dimanche est réservé aux loisirs et au culte catholique. L'action de Collinot s'inscrit ainsi dans une réflexion globale propre au $\mathrm{xIX}^{\mathrm{e}}$ siècle dans laquelle les dimensions professionnelle et morale s'entremêlent.

Collinot remporte une médaille de bronze et la mention «Honorable» en tant qu'institution ayant «contribué à la condition morale et matérielle des enfants employés dans l'industrie», remises par l'Impératrice Eugénie en $1868^{48}$. Plus tard, il reçoit le titre de chevalier de la Légion d'honneur pour ses actions en faveur de l'industrie et de l'amélioration des conditions morales et sociales de ses jeunes employés (1874). Bien que les actions sociales des fabriques citées dans le Bulletin de la Société de protection des apprentis et des enfants des manufactures soient nombreuses, les initiatives telles que celle de Collinot dans le secteur de la céramique restent rares. Peu de fabricants de céramique sont membres de la Société ou même cités dans les rapports. Parmi les usines qui contribuent à la condition matérielle des jeunes employés dans ces fabriques, citons les fabriques de Creil, de Choisy-le-Roi et celle d'Ivry. Collinot s'inspire peut-être de l'internat manufacturier d'Émile Muller (Ivry) ${ }^{49}$. Cet entrepreneur, orphelin lui aussi, permet aux orphelins ou aux jeunes employés de vivre dans l'internat, de disposer de salles d'études et de suivre des enseignements $^{50}$. La fabrique de Steinheill montre les mêmes problématiques que Collinot dans la formation et l'accueil des jeunes dans un internat ${ }^{51}$. Ces démarches d'éducation, de formation et d'éloignement de l'enfant de son univers familial semblent toutefois récurrentes à la fin des années 1860 . 
Collinot a compris que son intérêt pouvait nourrir les intérêts sociaux de son époque. La formation mutuelle d'apprentis fabriquant des œuvres commercialisables lui laisse la possibilité de poursuivre une production de chefsd'œuvre. Ses actions s'inscrivent dans un projet global de formation artistique au service de la revivification artistique industrielle.

\section{Enseigner le dessin et former les ouvriers des usines de céramique : nécessités et contradictions des entrepreneurs (1851-1881)}

Bien que l'apprentissage artistique soit réputé en crise depuis le $\mathrm{XVIII}^{\mathrm{e}}$ siècle - crise vraisemblablement amplifiée par l'interdiction des corporations par la loi Le Chapelier du 4 juin 1791 -, de nombreuses écoles et cours professionnalisants se développent grâce à des initiatives privées ou municipales durant la seconde moitié $\mathrm{du} \mathrm{xIX}^{\mathrm{e}}$ siècle $^{52}$. Le but de ces actions est de favoriser l'enseignement du dessin car il contribuerait à l'épanouissement de la culture générale des citoyens. La coopération Beaumont-Collinot s'inscrit dans ce contexte. La revivification de l'art industriel français à travers l'enseignement du dessin, rendu possible grâce à l'industrialisation des modèles et la création d'écoles d'art industriel sur le territoire national, devient un propos récurrent à cette époque. Cependant, l'enquête sur la situation des ouvriers et des industries d'art de $1881^{53}$ révèle que, malgré ces initiatives d'intérêt général, et malgré la création de l'Union centrale des beauxarts appliqués à l'industrie (la future UCAD) en 1864, les fabricants doutent des compétences techniques de leurs ouvriers et surtout du devenir de leur industrie. La lecture du rapport de cette enquête pose en fait la question de l'efficacité de l'enseignement artistique et de la formation en atelier, comme celui de Collinot. La commission d'enquête considère ces ateliers, s'inscrivant dans un «régime de patronage $\mathrm{e}^{54}$, comme l'héritage du système des corporations $\mathrm{d}^{\prime}$ Ancien Régime ${ }^{55}$. Ils estiment en outre que l'anonymat des auteurs de biens fabriqués ne leur permet pas de s'impliquer dans leur profession, les industries négligeant ainsi l'épanouissement de leurs ouvriers. En effet, ces derniers ne signent pas leurs productions en tant que collaborateurs. Selon les rapporteurs, ce manquement à l'émulation personnelle serait aussi causé par la division des tâches et la mécanisation des procédés de fabrication. En somme, la production massive serait néfaste à l'industrie car elle ne favoriserait pas la notion d'auteur. Dans les termes d'un débat très vif dans la seconde moitié du xIX ${ }^{\mathrm{e}}$ siècle, le multiple industriel annihilerait l'individualisation artistique.

Ce besoin de revalorisation de l'ouvrier-auteur est cependant confronté à une contradiction propre à de nombreuses entreprises: celle du recrutement et de la pérennisation des collaborateurs. 
Le cas de la faïencerie de Creil illustre ce paradoxe général: elle manque d'ouvriers qualifiés et souffre des problèmes de fonctionnement de l'école de dessin communale (1883). Cet établissement révèle un problème majeur des fabriques de province: le manque d'un programme défini. À ce propos, Stéphane Laurent souligne la complexité d'établir un enseignement artistique dans l'industrie: «À mi-chemin entre enseignement technique et enseignement artistique, il répond aux exigences d'un monde en pleine mutation, celui de la révolution industrielle, sans jamais perdre de vue une intention artistique $^{56}$.» L'échec est d'ailleurs amplifié par la désertion de ce cours de perfectionnement dispensé le soir par la commune en raison de la fatigue des enfants après leur journée de travail ${ }^{57}$. Ainsi, le directeur de Creil fait appel à des artistes parisiens pour réaliser des modèles et engage quelques graveurs et dessinateurs afin d'exécuter les dessins commandés. Ce sont ces derniers qui donnent quelques notions de dessin aux employés sur leur temps libre. Même si les commandes à ces artistes sont perçues comme nécessaires afin de renouveler les modèles, la mobilité des créateurs de modèles est également un problème pour la fabrique car celle-ci ne peut pas être complètement autonome: elle doit commander des modèles à des personnes extérieures à la fabrique qui peuvent proposer leurs créations à d'autres fabriques. Ainsi, la concentration locale d'ouvriers formés au dessin devient une nécessité aussi bien sociale qu'économique.

Cependant, une autre contradiction émerge. Alors que le patronat reconnaît la faible qualité de la formation de ses ouvriers, il émet quelques plaintes à propos de l'augmentation des salaires et surtout de celui des apprentis ${ }^{58}$. À Creil, les faïenciers adultes gagnent 10 francs par jour, les décorateurs moins de 7 francs par jour (le directeur de la faïencerie de Bordeaux évoque les mêmes chiffres dans sa déposition ${ }^{59}$ ). Les enfants gagnent environ 1,25 franc par jour, les femmes et les manœuvres 2 à 3 francs par jour. Cette hausse, l'abaissement des prix de vente, ainsi que la peur de la concurrence étrangère, deviennent des problèmes pour certains fabricants qui recrutent peu. Les fabricants peinent à trouver des apprentis correspondant à leurs attentes salariales. La commission désignée en 1881 pose encore, trente ans après le rapport de Laborde, la question du rôle de l'État dans la formation et dans l'enseignement global du dessin. Ces contradictions et nécessités ne trahissent-elles pas l'incapacité à définir les aptitudes globales des ouvriers des industries d'art? L'idéal serait de former selon les convenances des usines et de promulguer un enseignement général $\mathrm{du}$ dessin ${ }^{60}$. Selon les rapporteurs, certaines industries devraient être sous le joug de l'État, mais ce sont les initiatives privées et associatives qui doivent être l'objet d'une attention toute particulière. La création par l'État d'un petit nombre d'établissements-modèles permettrait de proposer une base commune, en complément du soutien des initiatives privées grâce à des subventions, à la mise à disposition d'enseignants, etc. Cette action étatique serait complétée par la multiplication de musées d'art industriel. Le but avoué est de tenter d'unifier les programmes d'enseignement. Les différents rapports des expositions universelles remettent également en question l'ensei- 
gnement général qui n'est pas soutenu par l'État de manière continue.

Les fours de l'entreprise du Parc des Princes ne s'éteignent pas à la mort de Collinot. Il semblerait que la fabrique soit en activité jusqu'en $1902^{61}$, peut-être sous la direction de son épouse. Pendant une vingtaine d'années, le technicien a formé de jeunes apprentis à une seule pratique artistique, celle des émaux en relief. Même si cette formation semble étroitement circonscrite, elle a le mérite de transmettre une pratique artistique dont l'esthétique est reconnue et appréciée du public. Dans cette entreprise, Collinot et Beaumont ont capitalisé leurs connaissances et concilié leur intérêt avec celui de leurs apprentis. L'action conjointe des deux hommes vise à revivifier la production céramique artistique. Dans l'anonymat de l'atelier, la formation des jeunes apprentis relève d'une démarche philanthropique revendiquée et saluée, leur projet s'inscrivant dans une dynamique générale, débutée plus d'un siècle auparavant, d'éducation indissociablement artistique et morale. Cependant, même si le transfert de compétences à travers cette formation mutuelle est une réussite - et ceci grâce à la transmission orale favorisée par cet «espace social ${ }^{62}$ » qu'est l'atelier -, on peut néanmoins se poser la question des limites de cette formation éminemment pratique. Ce problème est d'ailleurs identifié dans d'autres filières artistiques. À titre d'exemple, l'école de dessin industriel de Mulhouse souffrira de cette même stratégie ${ }^{63}$. Tous s'accordent sur le fait que l'État doit persister à encourager les initiatives locales et qu'il doit revaloriser l'enseignement artistique appliqué à l'industrie par la création de musées et d'écoles professionnelles. Toutefois, l'enquête de 1896, bien que confirmant l'utilité de la création des musées d'art industriel, montre que le manque d'ouvriers qualifiés fait encore partie des plaintes des fabricants. Il faudra attendre le début $\mathrm{du} x \mathrm{x}^{\mathrm{e}}$ siècle pour qu'un enseignement technique de la pratique céramique soit établi en lien avec tous ses acteurs $^{64}$.

\title{
Annexe 1: dépôt légal et descriptif des planches du Recueil pour l'art et l'industrie (1859-1873)
}

\author{
Source principale : http://artfl-project.uchicago.edu/
}

\section{4 décembre 1859, $n^{\circ} 2936$}

Gravés par Albert de Beaumont. "Ornements de diverses époques et de divers pays». 18 pl. Paris, imp. lith. \{Delâtre\}; \{Collinot\}. ENSEIGNEMENT.

\section{7 mars $1860, n^{\circ} 454$}

Porte du couvent des derviches au Kaire. - Couvercles de miroirs chinois. Tapis persan. - Vase chinois. - Cadre de miroir en bois sculpté, Bruges, seizième 
siècle. - Fontaine en marbre, kiosque de Bechiktash, Bosphore. Gravé par \{A. de Beaumont $\}$ et $\{$ Collinot $\}$. Paris, impr. en taille-douce \{Delâtre\}; \{E. Collinot\}.

\section{1 août 1861, no 1996}

Grilles en marbre d'un tombeau au Kaire. - Fontaine du kiosque de Hussein-Pacha sur le Bosphore. - Fenêtre en vitraux de couleur à Hispahan. Manuscrit arabe du onzième siècle. - Pot chinois. - Côté d'une cuirasse persane. - Dessins du Japon pour décors d'étoffe, laque, porcelaine. - Vase chinois. - Arcs de balustrade, balcon, niche, etc. - Frise de faïence persane. - Dessins japonais pour décors de paravent, etc. - Arcs de portes, fenêtres, etc. - Porte d'eau du palais ducal, Venise. - Fleurs de lis de toutes les époques et de tous les pays. zinc. - Dessins japonais pour décors. Lanterne et torchères du palais Strozzi. Cheminée de la salle des ambassadeurs, palais ducal, Venise. - Reliure persane. - Bible arménienne. - Pied de bronze du mât qui porte l'étendard de Chypre, à Venise. - Manuscrit persan. - Dessins japonais, etc. Gravé par \{A. de Beaumont $\}$ et $\{$ Collinot $\}$. Paris, impr. en tailledouce \{Delâtre\}. ENSEIGNEMENT.

\section{2 avril 1862, n 679}

34 pl. Gravé à l'eau-forte par \{Collinot\} et $\{$ A. de Beaumont\}. Paris, impr. en taille-douce \{Delâtre\}. ENSEIGNEMENT.

\section{3 mai 1863, no 1063}

Peinture japonaise-Porte de mosquée. - Fleurettes indo-persanes. - Animaux chinois et japonais. - Armes diverses. Étude de marguerites, gravure chinoise. - Formes diverses des cristallisations de la neige. - Carreau de faïence. - Trésor de Varsovie, vases divers. - Rosaces pour plafond. - Croquis japonais. - Fontaine à Constantinople. - Décoration en mosaïque de verre. - Page d'un manuscrit persan. - Une branche d'Altchoea, aquarelle chinoise. - Bordure en argent repoussé. - Porte à Ispahan. - Décoration d'un coffre en laque du Japon. - Dessins japonais pour décors. - Décoration des jardins du sérail. - Bas-reliefs de la scala d'oro, Venise. - Vases chinois. Chandelier arabe en bronze. - Papillons chinois. - Bijoux anciens. - Combinaisons de lignes géométriques. - Croquis de paysage japonais. - Grille en pierre d'une fenêtre à Diarbékir. - Marteau de porte du palais Pisani. - Idem, Campo San Maurizio. - Ornement sculpté sur un tombeau mogol. - Dessin décoratif chinois. - Reliure d'un manuscrit appartenant à l'ambassadeur de Perse. - Atrio d'un palais, Gênes. - Moitié d'un plat en laque de Perse. - Panneau de faïence dans la mosquée royale d'Ispahan. - Quatre panneaux japonais. - Plafond de la salle des Perles. Gravé par \{A. de Beaumont\} et $\{$ Collinot\}. Paris, impr. en taille-douce \{Delâtre\}. ENSEIGNEMENT.

\section{0 septembre $1865, n^{\circ} 1650$}

pl. 163, 165, 167, 171, 172, 173, 179 et 180, par \{A. de Beaumont\}. Paris, imp. \{Lemercier\}; \{de Beaumont\}. INDUSTRIE. 


\section{4 octobre $1865, n^{\circ} 1725$}

par \{Beaumont\}. Planches 164, 166, $168,169,170,174$ (175) à 178. Paris, imp. \{Lemercier\}. INDUSTRIE.

\section{3 novembre $1866, n^{\circ} 1826$}

Bassin des tilleuls, environs de Constantinople. - Bijoux russes. - Cadre italien. - Support en bois sculpté. - Carreau de faïence de Yali Kiosk. - Chapiteaux persans, cristallisations, cordons sculptés. - Galerie pyramidale et dôme d'un tombeau des kalifes. - Coupe en verre émaillé. - Dessin japonais sur un éventail. - Détails de cuirasses byzantine et persane. - Détails d'ornementation à la mosquée d'Omar. - Manuscrit arabe. - Oiseaux japonais. - Vases arabes et persans. Gravé par \{A. de Beaumont\}. Paris, imp. \{Lemercier\}. INDUSTRIE.

\section{2 décembre 1866, $\mathrm{n}^{\circ} 1985$}

Dessin du Japon. - Minaret d'Ibrahim agha comparé à l'Astrée polygonale (Madrépore). - Panneau de faïence. - Un puits à Venise. Gravé par $\{\mathrm{A}$. de Beaumont\}. Paris, imp. \{Lemercier\}. INDUSTRIE.

\section{7 juillet $1867, n^{\circ} 1119$}

Blasons divers. - Carquois persan. - Coffrets de l'Inde en bois de santal sculpté. - Dessins japonais. - Détails d'ornements, mosquée royale d'Ispahan. - Fleurs chinoises. - Fleurs du Japon. Fontaine d'Achmet, Constantinople. Grotte de Guzeldjeh, mer Noire. - Lettre du manuscrit de Sénèque, bibliothèque du Vatican. - Intérieur de la mosquée El Goury, Kaire. - Lions assyriens, cartha- ginois, sassanides et persans. - Morceau d'une frise peinte au palais de Nankin. Pêcherie dans les lagunes, Venise. - Porte de la mosquée de Soliman. - Porte du palais Van Axel, Venise. - Reliure vénitienne, manuscrit du conseil des Dix. - Une cour à Venise. Gravé par \{A. de Beaumont\}. Paris, imp. \{Lemercier\}. INDUSTRIE.

\section{5 août $1868, n^{\circ} 1366$}

Bouquets d'œillets rouges et or semés sur soie écrue de l'Inde. - Pacha de Trébizonde. - Les Jeux des enfants chinois. - Détails de la chaire de la mosquée el Teyloun (Kaire). - Détails de manuscrits indo-persans. - Carquois persan en cuir découpé et piqué sur velours rouge. Reliures persanes. - Détails du manuscrit de la Divine Comédie. - Venise. - Frise d'un palais à Nankin. - Plaque en bronze d'une serrure à Ispahan. - Frise sculptée d'un tombeau au Kaire. - L'amateur de fleurs. - Fragment du grand Koran de la mosquée El Azar, Kaire. - Dessin d'une bordure de faïence persane. - Carreau de faïence (Perse). - Aquarelle japonaise. - Dessin japonais. - Dessin du Japon. Caractères arabes de différents styles. Gravé par \{A. de Beaumont\}. Paris, imp. \{Lemercier\}. INDUSTRIE.

\section{5 octobre $1873, n^{\circ} 1722$}

Par \{Collinot\} et \{de Beaumont (15 pl.) Paris, imp. lith. \{Lemercier et Ce\}. INDUSTRIE. 


\section{Annexe 2: souscription du Recueil pour l'art et l'industrie par l'État, 1864-1870}

Le premier dépôt est effectué en 1864 et concerne les planches 1 à 162 . Un an après, le second dépôt met à disposition les planches 163 à 180 aux écoles. Entre l'année 1866 et 1867, les planches 181 à 198, puis 199 à 216 sont envoyées. L'année 1868 est le dernier envoi officiel, jusqu'à la planche 234 car, en 1870, le ministère se charge d'envoyer les planches manquantes de l'envoi des années précédentes

Source principale: base Arcade (base de données des Archives nationales) retraçant l'histoire des oeuvres d'art acquises par l'État français http:// www.culture.gouv.fr/documentation/ arcade/pres.htm

\begin{tabular}{|l|l|l|l|}
\hline \multicolumn{1}{|c|}{ Cote } & \multicolumn{1}{|c|}{ Destination } & \multicolumn{1}{c|}{ Nom de l'école } & Commentaires \\
\hline $\begin{array}{l}\text { AN, F/21/333, } \\
\text { dossier 4 }\end{array}$ & Marseille & école des Beaux-arts & \\
\hline $\begin{array}{l}\text { AN, F/21/334, } \\
\text { dossier 23 }\end{array}$ & Caen & $\begin{array}{l}\text { école gratuite de } \\
\text { dessin }\end{array}$ & \\
\hline $\begin{array}{l}\text { AN, F/21/343, } \\
\text { dossier 37 }\end{array}$ & Dijon & $\begin{array}{l}\text { école impériale des } \\
\text { Beaux-arts }\end{array}$ & \\
\hline $\begin{array}{l}\text { AN, F/21/359, } \\
\text { dossier 23 }\end{array}$ & Bordeaux & école de peinture & \\
\hline $\begin{array}{l}\text { AN, F/21/359, } \\
\text { dossier 23 }\end{array}$ & Bordeaux & $\begin{array}{l}\text { école gratuite de } \\
\text { dessin }\end{array}$ & \\
\hline $\begin{array}{l}\text { AN, F/21/359, } \\
\text { dossier 24 }\end{array}$ & Bordeaux & $\begin{array}{l}\text { école gratuite de } \\
\text { sculpture }\end{array}$ & \\
\hline $\begin{array}{l}\text { AN, F/21/386, } \\
\text { dossier 18 }\end{array}$ & Lunéville & $\begin{array}{l}\text { école gratuite de } \\
\text { dessin }\end{array}$ & \\
\hline $\begin{array}{l}\text { AN, F/21/386, } \\
\text { dossier 18 }\end{array}$ & Lunéville & $\begin{array}{l}\text { école industrielle et } \\
\text { gratuite de dessin }\end{array}$ & \\
\hline $\begin{array}{l}\text { AN, F/21/386, } \\
\text { dossier 27 }\end{array}$ & Nancy & $\begin{array}{l}\text { école gratuite de } \\
\text { dessin }\end{array}$ & \\
\hline $\begin{array}{l}\text { AN, F/21/393, } \\
\text { dossier 8 }\end{array}$ & Roubaix & $\begin{array}{l}\text { école gratuite de } \\
\text { dessin }\end{array}$ & \\
\hline $\begin{array}{l}\text { AN, F/21/393, } \\
\text { dossier 21 }\end{array}$ & Tourcoing & école de dessin & \\
\hline $\begin{array}{l}\text { AN, F/21/393, } \\
\text { dossier 27 }\end{array}$ & Valenciennes & $\begin{array}{l}\text { école industrielle et } \\
\text { gratuite de dessin }\end{array}$ & \\
\hline $\begin{array}{l}\text { AN, F/21/393, } \\
\text { dossier 27 }\end{array}$ & Valenciennes & $\begin{array}{l}\text { école gratuite de } \\
\text { dessin }\end{array}$ & \\
\hline $\begin{array}{l}\text { AN, F/21/405, } \\
\text { dossier 37 }\end{array}$ & Strasbourg & $\begin{array}{l}\text { école gratuite de } \\
\text { dessin }\end{array}$ & \\
\hline $\begin{array}{l}\text { AN, F/21/405, } \\
\text { dossier 37 }\end{array}$ & Strasbourg & $\begin{array}{l}\text { école industrielle et } \\
\text { gratuite de dessin }\end{array}$ & \\
\hline
\end{tabular}




\begin{tabular}{|l|l|l|l|}
\hline \multicolumn{1}{|c|}{ Cote } & \multicolumn{1}{|c|}{ Destination } & \multicolumn{1}{c|}{ Nom de l'école } & \multicolumn{1}{c|}{ Commentaires } \\
\hline $\begin{array}{l}\text { AN, F/21/406, } \\
\text { dossier 19 }\end{array}$ & Mulhouse & école de dessin & \\
\hline $\begin{array}{l}\text { AN, F/21/407, } \\
\text { dossiers 12 et 27 }\end{array}$ & Lyon & $\begin{array}{l}\text { école de dessin La } \\
\text { Martinière }\end{array}$ & $\begin{array}{l}\text { affectation: Société } \\
\text { d'enseignement pro- } \\
\text { fessionnel du Rhône }\end{array}$ \\
\hline $\begin{array}{l}\text { AN, F/21/415, } \\
\text { dossier 23 }\end{array}$ & Rouen & $\begin{array}{l}\text { académie de dessin et } \\
\text { de peinture }\end{array}$ & école de modelage \\
\hline $\begin{array}{l}\text { AN, F/21/430, } \\
\text { dossier 21 }\end{array}$ & Limoges & $\begin{array}{l}\text { placée sous le patro- } \\
\text { nage de la Société } \\
\text { d'agriculture de } \\
\text { Limoges }\end{array}$ \\
\hline $\begin{array}{l}\text { AN, F/21/430, } \\
\text { dossier 21 }\end{array}$ & Limoges & $\begin{array}{l}\text { école gratuite de } \\
\text { dessin }\end{array}$ & $\begin{array}{l}\text { placée sous le patro- } \\
\text { nage de la Société } \\
\text { d'agriculture de } \\
\text { Limoges }\end{array}$ \\
\hline
\end{tabular}

\section{Notes}

1. Sabine Pasdelou est doctorante en histoire de l'art à l'université Paris Ouest Nanterre La Défense. Ses recherches portent sur la production et la réception des céramiques japonisantes sur le territoire français entre 1861 et 1939 . Commissaire de l'exposition Vous avez dit japonisme?, auteure du catalogue (musée de Creil, 2010), elle a publié un numéro sur Le goût pour les caricatures de Grandville dans le décor imprimé sur assiettes à Creil, Choisy, puis Montereau (Les Amis de la faïence fine, 2012). Contact: sabine.pasdelou@gmail.com

2. Léon de Laborde, De l'union des arts et de l'industrie. Rapport sur les beaux-arts et sur les industries qui se rattachent aux beaux-arts, Paris, Imprimerie impériale, 1856.

3. Jean-François Luneau, «Art et industrie au $\mathrm{XIX}^{\mathrm{e}}$ siècle: des arts industriels aux industries d'art», dans Pierre Lamard et Nicolas Stoskopf (dir.), Art $\mathcal{E}$ industrie XVIII ${ }^{e}-\mathrm{XXI}^{e}$ siècle, Paris, Picard, 2013, p. 17-24.

4. Voir la série d'aquarelles et de dessins citée dans le Catalogue des aquarelles et des dessins par feu Adalbert de Beaumont. Faïences, costumes, bronzes et objets divers [Vente, Hôtel Drouot, Paris, 2 et 3 juin 1882]. Ses carnets de voyages ont permis d'établir la chronologie de ses déplacements. Au sujet de l'orientalisme de Beaumont, Rémi Labrusse (dir.), Islamophilies : l'Europe moderne et les arts de l'islam, Paris, Somogy, 2011, et Purs décors? Arts de l'Islam, regards du XIX ${ }^{e}$ siècle: collections des Arts décoratifs, Paris, les Arts décoratifs / Musée du Louvre éd., 2007.
5. Adalbert de Beaumont, "Salon de 1853», Le Nouveau Journal des théâtres, 9 juin 1853, p. 3.

6. Christophe Charle, Le siècle de la presse : 1830 1939, Paris, Seuil, 2004.

7. Philippe Burty, Chefs d'œuvre des arts industriels, Paris, Paul Ducrocq, 1866, p. 169. Voir Florence Slitine (dir.), Sèvres, Boulogne-Billancourt: la céramique indépendante, Luxembourg, Argusvalentines, 2007, p. 74.

8. Chantal Bouchon, «Adalbert de Beaumont (Paris 1809-Boulogne 1869). Du Cap nord à l'aventure céramique», Sèvres, $\mathrm{n}^{\circ} 12,2003$; Jacques Peiffer, Émaux d'Istanbul à Longwy: l'Europe de la faience, Thionville, Gérard Klopp, 1995; Florence Slitine (dir.), Sèvres, Boulogne-Billancourt..., op. cit.

9. Nous utilisons diverses archives telles que la correspondance de Beaumont, les archives relatives aux attributions du Recueil dans les écoles de province, le dossier de proposition pour la Légion d'honneur de Collinot, ainsi que la littérature contemporaine. Il ne reste aucun document issu de l'école de Collinot.

10. Il donne dans la presse des «articles sur des questions d'art; sa compétence en la matière lui vaut, sous l'Empire, d'être nommé membre du jury de peinture à plusieurs salons annuels et à 1'Exposition universelle de 1855 ", La Grande Encyclopédie. Inventaire raisonné des sciences, des lettres et arts, Paris, H. Lamirault, 1885-1902, t. V, p. 1050.

11. Adalbert de Beaumont, «De l'union des arts et de l'industrie par le comte de Laborde», $L^{\prime}$ Illustration, t. I, n 738 , avril 1857, p. 246-247.

12. Il publie notamment deux articles dans la Revue des deux mondes: "Les arts décoratifs en Orient et en France: les Gobelins», 15 octobre 
1861, p. 924-956, et «Les arts décoratifs en Orient et en France: Sèvres», 15 juin 1862, p. 804-840.

13. Voir Georg Maag, «Les machines ne sont rien sans l'art. De l'union des arts et de l'industrie du comte de Laborde, et les réactions de la presse», Romantisme, 1983, n ${ }^{\circ} 11$, p. 41-56.

14. Adalbert de Beaumont, "De l'union des arts et de l'industrie par le comte de Laborde», op. cit., p. 246-247.

15. Adalbert de Beaumont, "Les arts industriels en France et l'Exposition de 1863 ", Revue des deux mondes, 15 octobre 1863, p. 988.

16. Lettre de Beaumont à Alexandre de MetzNoblat, datée du 4 mars 1845, AD Meurthe-etMoselle, 52/J/10.

17. Lettre de Beaumont à Charles Cournault datée de janvier 1859. Voir Emmanuel Hecre, Les Orients de Charles Cournault, Metz, Serge Domini, 2004, p. 148.

18. Pierre Sanchez, Dictionnaire des céramistes, peintres sur porcelaine, verre et émail, verriers et émailleurs... 1700-1920, Dijon, L'échelle de Jacob, 2005, t. 1. La liste des exposants est extraite du Bulletin $d u$ Progrès d'octobre 1864.

19. Palais de l'Industrie, Exposition des beauxarts appliqués à l'industrie, Union centrale des beauxarts appliqués à l'industrie, Paris, [s. éd.], 1863, p. 106.

20. P. Sanchez, Dictionnaire des céramistes..., op. cit.

21. Lettre de Beaumont à Cournault datée de septembre 1857. Voir Ch. Bouchon, «Adalbert de Beaumont (Paris 1809-Boulogne 1869). Du Cap nord à l'aventure céramique», op. cit., p. 40.

22. Lettre de Beaumont à Cournault datée du 5 septembre 1862 , ibid., p. 38.

23. Lettre de Beaumont à Cournault datée de janvier 1860, Emm. Hecre, Les Orients de Charles Cournault, op. cit., p. 162.

24. Lettre de Beaumont à Cournault datée de janvier 1859, ibid., p. 148.

25. Lettre de Beaumont à Cournault attribuable à l'hiver 1863, ibid., p. 177.

26. Adalbert de Beaumont et Eugène Collinot, Les recueils de dessins pour l'art et l'industrie, Paris, Delâtre, 1859-1873.

27. Agnès Lahalle, Les écoles de dessin au XVIII siècle. Entre arts libéraux et arts mécaniques, Rennes, Presses universitaires de Rennes, 2006.

28. Frits Lugt, Les marques de collection de dessins et d'estampes, Amsterdam, Vereenigde drukkerijen, 1921, p. 135.

29. Dominique Varry (dir.), Histoire des bibliothèques françaises. Les bibliothèques de la Révolution et du XIXesiècle, Paris, Promodis/Éditions du cercle de la librairie, 1991, t. III. p. 114.
30. Feuillet placé dans le dossier de proposition pour la Légion d'honneur de Collinot, AN, $\mathrm{F} / 12 / 5112$.

31. Lettre du maître des requêtes au Conseil d'État au ministre du Commerce datée du 19 octobre 1869, AN, F/12/5112.

32. Brevet $n^{\circ} 64600$ de Collinot, en date du 30 septembre 1864 .

33. Selon Collinot, cette appellation permet seulement la compréhension du résultat de cette technique. Ce type de peinture vitrifiée se compose d'un colorant céramique et du fondant, matériau vitrifiant qui est souvent composé d'alcalins, qui permettent à la couleur ainsi vitrifiée d'adhérer à la pièce. Nicole Blondel, Céramique, vocabulaire technique, Paris, Éditions du patrimoine, 2001, p. 34 et p. 254. C'est d'ailleurs Jacques Peiffer qui a donné cette appellation technique d'émaux en relief à décor cerné.

34. Cette association est reconnue d'utilité publique par un décret de Napoléon III, le 4 juillet 1868 .

35. Bulletin de la Société de protection des apprentis et des enfants des manufactures, Paris, A. Chaix et cie, 1867-1914.

36. La loi du 22 mars 1841 stipule que les enfants ne peuvent travailler avant l'âge de 8 ans. La loi du 22 février 1851 indique quant à elle qu'ils doivent être scolarisés jusqu'à l'âge de 12 ans. Voir Claire Lemercier, " "Il faut parler de ce qu'on sait". "Hommes pratiques", "économistes distingués" et législateurs face au travail des enfants (1837-1874)», dans Christophe Charle et Julien Vincent (dir.), La société civile: savoirs, enjeux et acteurs en France et en Grande-Bretagne, 1780-1914, Rennes, Presses universitaires de Rennes, 2011, p. 127-145.

37. Collinot obtient la Légion d'honneur en 1874, AN, F/12/5112.

38. Feuillet présentant Collinot, écrit par Beaumont le 18 juin 1867, AN, F/12/5112.

39. Paul Fouchet fait partie du comité d'enseignement artistique de la Société.

40. Lettre de M. Guiral, curé de la paroisse Notre-Dame de Boulogne, au ministre du Commerce, datée du 16 septembre 1873, AN, $\mathrm{F} / 12 / 5112$.

41. Bulletin de la Société..., op. cit., vol. 2, p. 272.

42. Michel Chalopin, L'enseignement mutuel en Bretagne. Quand les écoliers bretons faisaient la classe, Rennes, Presses universitaires de Rennes, 2011; Bruno Poucet, "Petite histoire de l'enseignement mutuel: 1 'exemple du département de la Somme", Carrefours de l'éducation, n² 27, 2009/1, p. 7-18; Raymond Tronchot, L'enseignement mutuel en France de 1815 à 1833, les luttes politiques et reli- 
gieuses autour de la question scolaire, thèse de doctorat d'État ès lettres, 1973.

43. Bulletin de la Société..., op. cit., vol. 2, p. 272.

44. Noël Régnier, L'industrie française au XIX ${ }^{e}$ siècle, Paris, Léon Sault, 1878, p. 418.

45. Lettre de $M$. Guiral au ministre du Commerce, datée du 16 septembre 1873, AN, $\mathrm{F} / 12 / 5112$.

46. Adèle de Chambry, "Le monde de la mode», Le Feuilleton de la Presse, 26 septembre 1880, n 266, [p. 3].

47. Bulletin de la Société..., op. cit., vol. 2, p. 182.

48. Feuillet dans le dossier de proposition pour la Légion d'honneur de Collinot, AN, F/12/5112.

49. Jean-François Belhoste, «Émile Muller (1823-1889), ingénieur alsacien, promoteur de la céramique décorative", dans P. Lamard et N. Stoskopf (dir.), Art \& industrie XVIII'-XXI siècle, op. cit., p. 191-200.

50. Bulletin de la Société..., op. cit., vol. 1, p. 417.

51. Ibid., p. 305.

52. Ces initiatives associatives ou privées sont soutenues dans les rapports des expositions universelles entre 1851 et 1867. Voir Renaud d'Enfert, L'enseignement $d u$ dessin en France: figure humaine et dessin géométrique (1750-1850), Paris, Belin, 2003, et Stéphane Laurent, L'art utile: les écoles d'arts appliqués sous le Second Empire et la Troisième République, Paris, L'Harmattan, 1998, et Les arts appliqués en France: genèse d'un enseignement, Paris, CTHS, 1999.

53. Cette enquête a été instituée par le ministère de l'Instruction publique et des Beaux-Arts le 24 décembre 1881; son rapport a été dirigé par Antonin Proust: Commission d'enquête sur la situation des ouvriers et des industries d'art, Paris, A. Quantin, 1884.

54. Ibid., p. VI et IX.

55. Steven L. Kaplan et Philippe Minard (dir.), La France, malade du corporatisme? XVIII $-\mathrm{XX}^{e}$ siècles, Paris, Belin, 2004.

56. S. Laurent, L'art utile..., op. cit., p. 17.

57. Commission d'enquête..., op. cit., p. 381.

58. Ibid., p. 380.

59. Ibid., p. 385.

60. À ce sujet, on peut se référer au rapport de l'inspecteur général de l'enseignement du dessin au ministère de l'Instruction publique et des Beaux-Arts, Paul Colin, L'enseignement des arts $d u$ dessin, Exposition universelle de 1889, Beaux-Arts, extraits du Journal officiel du 5 novembre 1889, Paris, Impr. des Journaux officiels, 1889.

61. Jacques Peiffer, Émaux d'Istanbul à Longwy..., op. cit., p. 165.

62. Richard Sennett, Ce que sait la main. La culture de l'artisanat, Paris, Albin Michel, 2010, p. 104.

63. Bernard Jacqué, "Créer des motifs pour l'impression à Mulhouse au XIX siècle: la formation et ses limites", dans P. Lamard et N. Stoskopf (dir.), Art $\mathcal{E}$ industrie XVIII ${ }^{e}$-XXI siècle, op. cit., p. 133140.

64. Voir l'article de Florent Le Bot dans ce numéro. 